\title{
NEURAL NETWORK APPLICATION TO SHIP POSITION ESTIMATION
}

\author{
D.G. Lainiotis, K.N. Plataniotis, Dinesh Menon, C.J. Charalampous
}

\author{
Florida Institute of Technology
}

\section{MELBOURNE, FLORIDA}

\begin{abstract}
The real time estimation of ship motion is considered in this paper. The problem is viewed as an estimation/ prediction problem for partially unknown systems. A neural estimator based on a dynamic recurrent neural network is considered. The model that describes the ship motion dynamics is presented, and the neural algorithm is tested and evaluated via extensive simulations. The results show that the new algorithm has excellent performance, and a significant saving in computational time is achieved.
\end{abstract}

\section{INTRODUCTION}

The accurate on-line estimation of ship motion is essential to many ship related problems such as ship steering [1], dynamic positioning [2], marine oil exploration [3], off shore platforms, and aircraft landing and take-off [4]. Based upon predicted ship motion, the necessary control commands for the control of highly qualified ships like hovercrafts are calculated and generated. Ship motion prediction is also essential when accurate control of position mechanisms for guns or missiles is required. When tracking of a maneuvering target is the objective, there is a considerable amount of delay in transmitting information regarding the motion to the position mechanism. It is obvious that accurate predictions are required before an appropriate tracking command can be issued [3].

In the past a lot of studies have been carried out for the solution of the ship positioning problem, most of them utilizing the Kalman Filter approach, or other leastsquares estimation methods [1],[3]. However the design of a statistical estimator like the Kalman Filter requires the definition of a linear model describing the motion of the ship. More specifically, it requires a state-space representation of this motion. In most of the cases, the model equations are derived from the ship motion spectral density which corresponds to a particular seaship condition, the wave excitation input, and a gaussian random noise as driving input. In other words the seastate magnitude, the ship speed, the ship heading with respect to the waves, and the disturbance pattern of the sea waves are incorporated in the model $[5],[6]$.

In this paper the estimation of the ship motion based on a linear state-space model that includes the waveexcitation input is proposed. Specifically, the paper is organized as follows. In Section II, the general formulation of the ship motion dynamics is presented. In Section III, the neural solutions are tested in extensive simulations on the ship motion model, and are compared with the conventional methods. Conclusions are given in Section IV.

\section{SHIP MOTION DYNAMICS}

The motion of the ship can be described by a set of differential equations. As it was mentioned above, the model depends on the sea-state magnitude, the ship speed and the ship heading with respect to the waves. For a rigid ship travelling with a constant forward speed and in the direction which makes an arbitrary angle with regard to sinusoidal waves, its motion can be described by a set of second-order linear differential equations of the form:

$$
a \ddot{q}(t)+b \dot{q}(t)+c q(t)=\xi(t)
$$

where,

$q(t)$ represent a vector of surge, sway and heave motions or variations of roll, pitch and yaw orientations, $\xi(t)$ represents the sea-wave excitation, and $a, b, c$ are constants that represents the virtual mass, damping and restoring stiffness, and are determined by the dimensions and shape of the ship. 
The above linear model can be obtained using the power spectral density function under different sea-wave excitation [5]. The most important part of the equation is representation of the periodic sea-wave excitation. The wave excitation can be approximated by the superposition of sinusoidal waves [6].

$$
\xi(t)=\Sigma A_{i} \cdot \sin \left(\omega_{i} \cdot t+b_{i}\right)
$$

where, $A_{i}$ is the amplitude of wave excitation, $\omega_{i}$, the frequency and $b_{i}$, the phase of each different wave.

In most of the cases, the amplitude and the frequency of the waves are considered to be time-invariant. When the ship is moving with forward speed, there exists a certain relation between the actual wave frequencies, and the frequencies encountered by the ship[6]. Following this relation, the direct influence of the waves on the ship is given as follows:

$$
\begin{gathered}
\xi_{e}(t)=\Sigma A_{i} \cdot \sin \left(\omega_{e i} \cdot t+b_{i}\right) \\
\omega_{e i}=\omega_{i}-\frac{\omega_{i}^{2}}{g} \cdot v \cdot \cos (x)
\end{gathered}
$$

where, $\omega_{e i}$ is the transformed wave frequency, $v$ is the forward speed of the ship, $x$ is the angle between the ship heading and the wave direction, and $g$ is the standard gravitational acceleration.

Moreover since the energy of each individual wave component rapidly decreases as the frequency of the wave increases, the above expression can be simplified further. Truncating the high frequency components the expression takes the following form:

$$
\xi_{e}(t)=\sum_{i=1}^{N} A_{i} \cdot \sin \left(\omega_{e i} \cdot t+b_{i}\right)
$$

It is reported in numerous studies [1],[5], [6] [7] that value of 3 for $\mathrm{N}$, is sufficient to approximate the wave excitation input, in the case of small ships. After all the approximations and transformations, the equation for the ship motion in the equivalent state space representation can be written as follows:

$$
\begin{aligned}
& \dot{x}(t)=\left[\begin{array}{cc}
0 & 1 \\
-\frac{c}{a} & -\frac{b}{a}
\end{array}\right] \cdot x(t)+\left[\begin{array}{l}
0 \\
\frac{1}{a}
\end{array}\right] \cdot \xi_{e}(t)+w(t) \\
& z(k)=\left[\begin{array}{ll}
1 & 0
\end{array}\right] \cdot x(k)+v(k)
\end{aligned}
$$

where, $x(t)$ is the state vector which represents the ship motion. It is a $2 \times 1$ vector defined as follows:

$$
x(t)=\left[\begin{array}{l}
q(t) \\
\dot{q}(t)
\end{array}\right]
$$

$\xi_{\mathrm{e}}(\mathrm{t})$ is the truncated wave excitation input, $w(t)$ is the state random noise, $z(k)$ is the discrete measurement vector and $v(k)$ is a random noise which corrupts the measurements.

The position and velocity of the ship are usually measured by on-board sensors. Since all the states are not measurable, and the measurements always contain noise, filters are cmployed to estimate the actual position of the ship. The objective is to obtain the optimal, state estimate $\hat{x}(\mathrm{k} / \mathrm{k})$ of the state $x(\mathrm{k} / \mathrm{k})$, in the mean square sense, given the measurement record, $\mathrm{Z}(\mathrm{k})=\{z(1), z(2), \ldots, z(\mathrm{k})\}$.

\section{SHIP POSITION ESTIMATORS}

In the past Kalman Filter based techniques, or other statistical filters have been used in connection with state space models in order to provide meaningful and accuratc estimates of the ship motion. If the sea-wave excitation (sea condition) is known in advance, and all other dynamic and statistical specifications of the above model meet the assumptions of the Kalman Filter, then this filter is the optimal estimator and provides the most accurate estimate. However the sea condition is not always known in advance to the designer of the filter. In this situation, when a mismatch occurs between the actual model and the model used by the designer, the Kalman filter fails.

A more robust statistical filter, namely the Adaptive Lainiotis Filter [8],[9], was successfully used in this situation. Unlike the Kalman Filter, this new filter, due to its adaptive nature, identifies in real time, the actual model and provides the appropriate solutions [8],[11]. Recently, neural networks have been used to estimate 
states of dynamic systems [12],[13],[14]. In this paper, a neural network is used to provide the ship motion estimates. The estimator is a multilayer recurrent neural network, trained using back-propagation technique, to provide estimates of the ship position.

The following simulation experiments are performed:

\section{SIMULATION I:}

In this experiment, the neural estimator is compared with the optimal Kalman filter. It is supposed for comparison purposes that the dynamic model is completely known, and the statistical Kalman filter is matched to the actual dynamic and statistical model of the ship dynamics. Although this situation is highly unrealistic, this comparison is made to evaluate the performance of the neural estimator, viz. a viz., the inaccessible performance of the optimal Kalman filter. The experimental set-up is given below:

\section{- System Model:}

$$
\begin{aligned}
\dot{x}(t) & =\left[\begin{array}{cc}
0 & 1 \\
-0.36 & -0.06
\end{array}\right] \cdot x(t)+\left[\begin{array}{l}
0 \\
1
\end{array}\right] \cdot \xi_{e}(t)+w(t) \\
z(k) & =\left[\begin{array}{ll}
1 & 0
\end{array}\right] \cdot x(k)+v(k) \\
& \xi_{e}(t)=\sum_{i=1}^{3} a_{2 i-1} \cdot \sin \left(\omega_{i} \cdot t\right)+a_{2 i} \cdot \cos \left(\omega_{i} \cdot t\right)
\end{aligned}
$$

where,

$a_{2 i-1}=A \cdot \cos \left(b_{i}\right), a_{2 i}=A \cdot \sin \left(b_{i}\right)$. The amplitude of the wave excitation input, $A=0.5$, with a constant excitation frequency, $\omega_{i}=\pi / 4$; different phases are, $b_{1}=2 \pi / 3$, $b_{2}=\pi / 3, b_{3}=\pi / 6 . \quad Q=0.25$ and $R=0.1$, are zero-mean, white, gaussian, plant and measurement noise covariances, respectively.

In order to estimate the states of the above model, the following estimators are used in this first experiment:

\section{Statistical estimator: Kalman filter}

The Kalman Filter is matched to the above dynamic and statistical model. All the quantities of the state-space model is assumed to be known a-priori to the filter designer. Moreover, the filter starts its recursive cycle assuming initial state estimate, $x(0 / 0)=0$, and initial covariance, $P(0 / 0)=100$.

\section{Neural estimator: recurrent, multilayer network}

— network topology:

- two input nodes: the current and the previous neural output are used as input signals

- two output nodes: the estimates of the system states. The neural network has so many output nodes, as the states of the model

- two hidden layers with $10-10$ hidden nodes respectively

- learning parameters:

- learning rate: 0.005 , momentum: 0.2

- Training procedure:

- backpropagation training algorithm

- the network knows the actual states of the model during the training phase. The target vector is the actual state vector

- the network tries to minimize the square error between the current output and the target vector

- The training data set is produced by running the system equations. The training set consists of 150 input/output pairs $(z(k), x(k))$

- The test data record consists of a sequence of data points produced separately from the training record

- the training procedure is terminated if the training error tolerance is less than 0.01 or if the number of iterations of the training set is more than 5000

\section{Observations:}

In Figs.1-3, the performance of the Kalman Filter in the estimation of the position and the normalized error over 100 Monte Carlo trials are given. The average error in the performance during the Monte Carlo simulation is calculated using the following performance index:

$$
M S E=\frac{1}{m c} \cdot \sum_{i=1}^{m c}\{x(k)-\hat{x}(k / k)\} /(x(k))
$$

From the figures it is obvious that the Kalman Filter 
performs very well, when all the parameters of the dynamic and statistical model are known. In Figs. 2 and 3 , the performance of the neural estimator in the estimation of the ship position and its error over 100 Monte Carlo runs, using the same performance index shown above, is given. In summary, the following conclusions can be drawn:

- for a linear, gaussian model, if the underlying statistics and dynamics of the phenomenon is completely known to the estimator, the Kalman filter provides the optimal results.

- the neural estimator performs similar to the optimal filter even though it is a nonlinear structure applied to a linear, gaussian model.

\section{SIMULATION II:}

It was mentioned in the introduction that many times it is more important to predict ahead the position of the ship, rather than estimate its current position. This will be the subject of the second experiment. In particular, we are interested in comparing the performance of a neural predictor, with that of the Kalman predictor. The model used in the experiment is the same twodimensional state-space model used in the previous one. The following two estimators / predictors are compared:

\section{Statistical predictor: Kalman filter}

Two Kalman filters are used in this simulation experiment. The first one is matched to the actual dynamic model. All the quantities of the dynamic model is supposed to be known to the designer. The second Kalman filter does not know the exact dynamic model. More specifically, in its design another variation of the wave excitation has been assumed. Both the filters assume that the measurement noise is gaussian, zero mean, with variance, $R=0.1$. The initial state is assumed to be gaussian, with the initial estimate, $\hat{x}(0 / 0)=0$, and initial covariance, $P(0 / 0)=100$. The objective for each of them is the prediction of the ship position two seconds ahead.

\section{Neural predictor: recurrent multilayer network}

- network topology:

- five input nodes: the current and the four previous measurements are used as input signals
- one output node: the prediction of the next measurement value

- three hidden layers with 10-10-10 hidden nodes respectively

- learning parameters:

- learning rate: 0.001 , momentum: 0.1

- Training procedure:

- backpropagation training algorithm

- the network does not know the actual states of the model during the training phase. The target vector is again a measurement value

- the nctwork tries to minimize the square error between the current output and the target vector

- the test data record consists of a sequence of data points produced separately from the training record

- the training procedure is terminated if the training error tolerance is less than 0.01 or if the number of iterations is more than 5000

\section{Observations:}

As was expected, the neural predictor performs better than the mismatched Kalman predictor, despite the minimal information required for its training. The prediction of the network, is shown in Fig. 4. However when the model that describes the wave excitation is completely known, the Kalman filter provides the optimal predictions. The performance of the two Kalman predictors and the corresponding errors over 100 Monte Carlo runs are given in Figs. 5-8. These results lead us to the following conclusions:

- the neural predictor requires very little information about the nature of the model, and provides best predictions, with total ignorance of the actual dynamic and statistical model.

- the statistical predictor (Kalman filter) does not perform as well, and provides suboptimal solutions when a mismatch between the actual model and the one used in the design exist. The comparative graphical representations, shown in Figs. 7 and 8 confirm the above conclusions. 


\section{CONCLUSIONS}

The real time ship motion estimation and prediction was considered in this paper. The approach taken, was to design a neural network based estimator, that can handle more realistic scenarios about the underlying physical model. A comparison with the most widely used statistical Kalman filter estimator, was made. Simulation experiments were carried out in order to assess the performance. In the ship motion prediction problem, the neural network predictor shows excellent performance, though it was derived using minimal information about the dynamics of the model.

\section{REFERENCES}

[1] R.E. Reid, A.K. Tugcu, B.C. Mears, "The use of wave filter design in Kalman Filter state estimation for the automatic steering problem of a tanker in a seaway", IEEE Transactions on Automatic Control, Vol. AC-20, No. 7, pp. $917-$ 922, 1984.

[2] T. M. Weiss, T. W. Devries, "Ship motion measurement filter design", IEEE Journal of Ocean Engineering, Vol. OE-2, No. 4, October 1977, pp. 325-330.

[3] M.S. Triantafyllou, M.Bodson, M. Athans, "Real time estimation of ship motion using Kalman Filtering technique", IEEE Journal of Ocean Engineering, Vol OE-8, pp. 8-20, January 1983.

[4] M.M. Siolar, B.F. Doolin, "On the feasibility of real time prediction of aircraft carrier motion at sea", IEEE Transactions of Automatic Control, Vol AC-28, pp. 350-356, March 1983.

[5] R. Bhattacharyya, "Dynamics of Marine Vehicles", Wiley N.Y., 1978.

[6] J.C. Chung, Z. Bien, Y.S. Kim, "A note on shipmotion prediction based on wave-excitation input estimation", IEEE Journal of Ocean Engineering,
Vol. OE-15, No. 3, July 1990, pp. 244-250.

[7] B. Friedland, "Estimating angular velocity from output of rate integrating syro", IEEE Transactions on AES-11, July 1975, pp. 551-555.

[8] D.G. Lainiotis et al., "Real time ship motion estimation using Lainiotis Filters", IFAC Workshop, CAMS-'89, Expert Systems in Marine Automation, pp. 293-301, 1989.

[9] D.G. Lainiotis, "Optimal adaptive estimation: Structure and parameter adaptation", IEEE Transactions on Automatic Control, Vol. AC-16, pp. 160-170, April 1971.

[10] D.G. Lainiotis, "Partitioning: A unifying framework for adaptive systems, I-Estimation", Proceedings of IEEE, Vol. 64, pp. 1126-1142, August 1976.

[11] D.G. Lainiotis, C.J. Charalampous, P. Gianakopoulos, S. Katsikas, "Real time ship motion estimation", Proceedings Oceans '92, pp. 283-287, Newport, Rhode Island, 1992.

[12] D.E. Rumelhart, J.L. McClelland, "Parallel Distributed Processing: Explorations into the Microstructure of Cognition, Vol. I", M.I.T. Press, 1986.

[13] J.P. De Gruyenaece, H.M. Haffer, "A comparison between Kalman Filters and recurrent neural networks", Proceedings of IJCNN-92, Vol. IV, pp. 247-251, 1992.

[14] A.J. Kanekar, A. Feliachi, "State estimation using artificial neural networks", Proceedings of IEEE Systems and Engineering Conference, pp. $552-$ $556,1990$. 


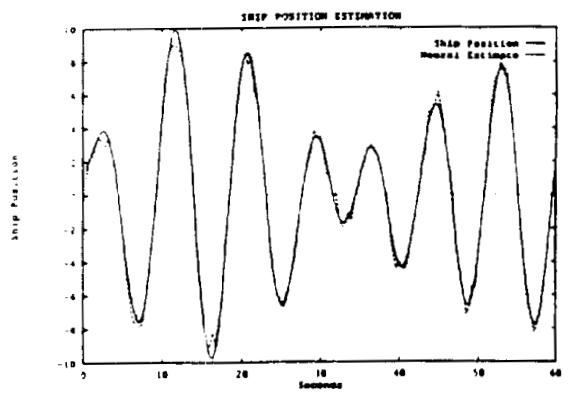

Fig. I Ship position estimation: neural estimator

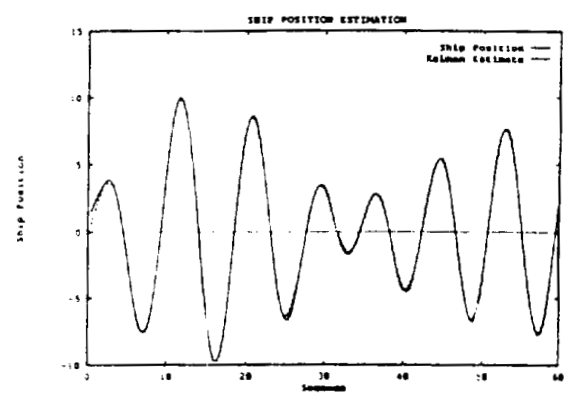

Fig. 2 Ship position estimation: Kalman filter

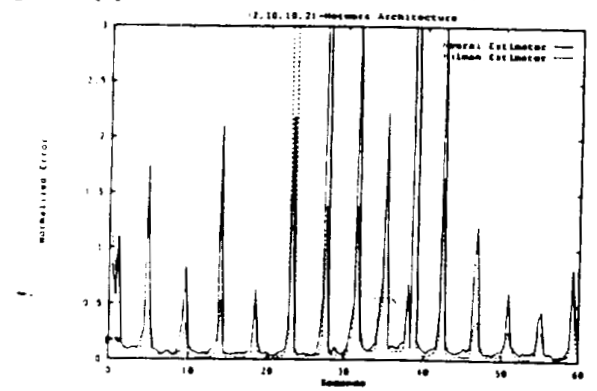

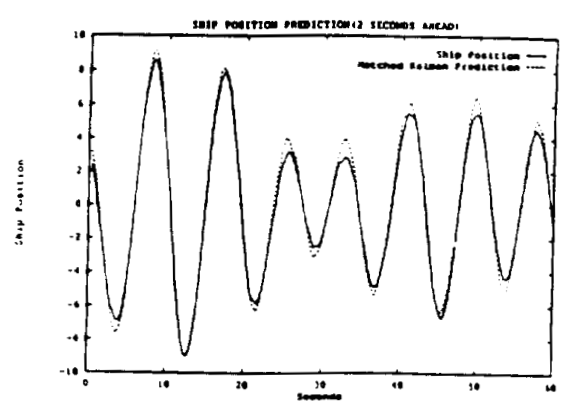

Fig. 5 Ship position prediction : Matched Kalman filter

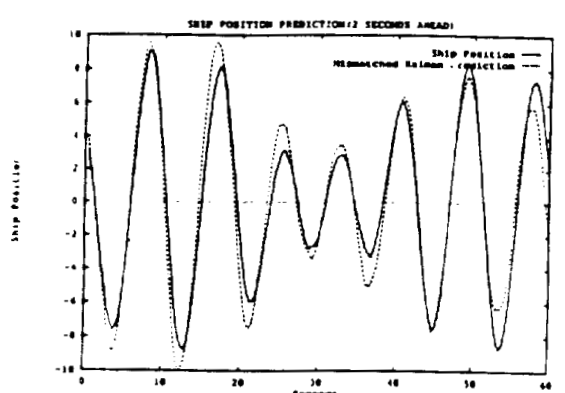

Fig. 6 Ship position prediction : Mismatched Kalman filter

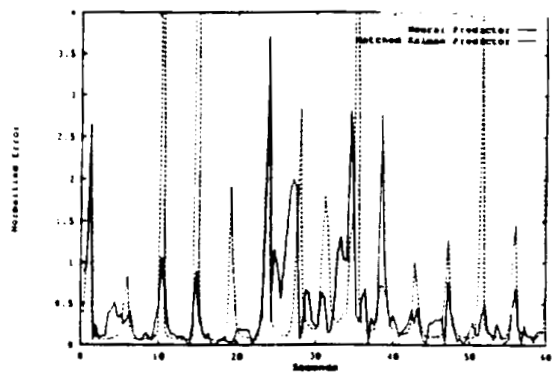

Fig. 3 Estimation, Normalized error. Comparative evaluation, 100 MC Fig. 7 Prediction,Normalized error: Comparative evaluation 100 MC

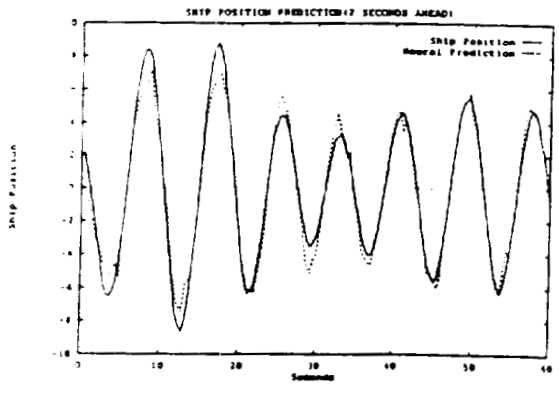

Fig. 4 Ship position prediction: neurai predictor

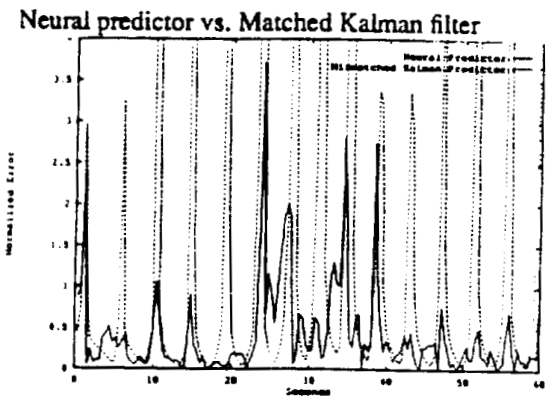

Fig. 8 Prediction,Normalized error: Comparative evaluation $100 \mathrm{MC}$ Neural oredictor vs. Mismatched Kaiman filter 


\section{DEVELOPMENT OF A SIX DEGREE OF FREEDOM BUOY DESIGN AND ANALYSIS PROGRAM WITH VALIDATING DATA}

\author{
W. A. Venezia \\ Ocean Systems Development Corp. \\ 375 N. W. 35th Lane \\ Boca Raton, Florida 33431 \\ (407) $451-7226$
}

\author{
A. M. Clark \\ Harbor Branch Oceanographic Inst. \\ 5600 Old Dixie Highway \\ Fort Pierce, FL 34946
}

(407) $465-2400$

\author{
K. F. Schmitt \\ Science Applications International Corp. \\ 10260 Campus Point Drive \\ San Diego, CA 92121
}

(619) $546-6369$

\begin{abstract}
A six degree of freedom buoy design and analysis program is given. The buoy program extends original work published by the Woods Hole Oceanographic Institution for roll and heave response of free floating axisymetric bodies.

The program predicts the probable amplitude of buoy displacement, velocity, acceleration, and jerk for heave, surge, and sway, motions and probable amplitude of roll, pitch, and yaw angular displacements, velocities, and accelerations. Given is an overview of the equations of motion, simplifying assumptions, and a description of the computational method.

The paper contributes limited verification of the computational method with a summary of computer predictions and experimentally obtained data on buoy motion. The data on motions is obtained from buoys designed with specific buoy motion requirements. Experimental data is given for various sea states and buoy types.
\end{abstract}

\section{INTRODUCTION}

Modern oceanographic measurement techniques often require highly stable platforms with predictable levels of motion[1,2]. Modern air sea interaction studies depend on at-sea experimental model verifications to test and evaluate emerging theories in a wide variety of ocean sciences. Experiments range from global heat flux, including prediction of momentum exchange in the presence of waves, to the understanding of radio wave propagation over the sea.

The advent of the personal computer ( $\mathrm{PC}$ ) provided design engineers with a computational machine that allows for rapid and convenient solution of many classical engineering problems. Off the shelf software is available to solve many of the typical problems encountered in fluid mechanics, solid mechanics and dynamics. Ocean engineers seeking to solve complex problems related to the ocean environment are developing their own personal computer based software to satisfy system requirements and solve design conflicts[3]. Typical programs include static and dynamic cable, buoy, and towed systems problem solvers. With an increasing number and variety of computer models available it is now more important than ever to have applicable, high quality, well understood data for validating emerging computer codes.
Accurate computer aided tools for the design and analysis of moored and free floating buoy systems require full scale validation and refinements until the user has confidence the program is generating realistic output.

Ocean Systems Development Corporation (OSDC) was founded to provide high quality ocean engineering products and services to the maritime industry. OSDC supports theoretical design studies leading to innovative oceanographic instrumentation and stabilized instrument platforms while providing experienced technical support personnel for the solution of practical problems related to the ocean environment

The OSDC six degree of freedom (6DOF) computer solution for free floating buoys presented here was developed over the past twelve years from ideas given in original work published by The Woods Hole Oceanographic Institution (WHOI)[4]. The WHOI reports present an analysis method and FORTRAN computational code designed to run on a main frame computer for the heave, roll, and pitch response of free floating bodies of cylindrical shape. We extended the work to include surge, sway, and yaw and now have a working code written in Microsoft QBasic for a PC. The program predicts the probable amplitude of displacement, velocity, acceleration, and jerk for heave, surge, and sway, motions and probable amplitude of roll, pitch, and yaw angular displacements, velocities, and accelerations.

We compare the output of the code to the response of three buoys built using the code as a design tool. The data we draw on is separate deployments. The deployments are; a $10 \mathrm{~m}$ spar at Lock Linnie, Scotland, a $27 \mathrm{~m}$ spar off Kauai, Hawaii ,and a $2 \mathrm{~m}$ diameter torridal buoy off Fort Pierce, Florida. The 6DOF buoy design and analysis program was used in each case to predict motions in the design cycle. In each case the buoy motion data was obtained from a strap down motion monitoring package onboard the buoy. Sea state spectral data corresponding to each set of buoy motion data was experimentally obtained from either a wave staff, a wave rider, or a subsurface pressure/water velocity sensor. To increasing extents, the predicted and measured buoy response is given. 